\title{
Interleukins As a Marker of Inflammation in Diabetic Foot Syndrome and Type 2 Diabetes Mellitus
}

\author{
(D) Süleyman KALELİ1, iD Ceyhun VARIM22, id Ahmet NALBANT2, iD Hayrullah YAZAR33, iD Mehmet AKDOĞAN3
}

1Sakarya University Faculty of Medicine, Department of Medical Biology, Sakarya, Turkey

2Sakarya University Faculty of Medicine, Department of Internal Diseases, Sakarya, Turkey

${ }^{3}$ Sakarya University Faculty of Medicine, Department of Medical Biochemistry, Sakarya, Turkey

\section{ABSTRACT}

Objective: Diabetic foot ulcers, one of the factors that determine the life time and quality of life in diabetic patients are one of the most frequent causes of hospitalization and surgery. In this study, we aimed to investigate the levels of interleukin (IL)-12 P70, 17, 18, hemoglobin (Hb) A1c, and some parameters in subjects with type 2 diabetic mellitus with diabetic foot and in healthy subjects.

Methods: The study was performed on three groups. They were patients with diabetic foot, with diabetes mellitus type 2 and healthy individuals (control). IL-12 P70, IL-17, and IL-18, HbA1c levels and other biochemical parameters were compared between three groups.

Results: We found higher IL-17, IL-18, white blood cells, glucose and HbA1c in the diabetic group and diabetic foot group than in the control group. IL-12 P70 levels in the diabetic foot group were significantly higher than the control group. The performances in diagnosing of IL-12 P70, 17 and 18 as a biomarker for diabetic foot were found statistically significant. IL-12 P70, 17 and 18 levels in diabetic patients were not different from the levels in patients with diabetic foot.

Conclusion: According to our results, IL-12 P70, IL-17 and IL-18 can be used as biomarkers for diabetic foot. Further studies are needed to show the role of IL-12 P70, IL-17 and IL-18 levels as a biomarker in chronic inflammatory diseases.

Keywords: Diabetic foot, diabetes mellitus, chronic inflammatory diseases, interleukins, biomarkers

\section{Introduction}

Diabetes mellitus (DM) type 2 is the most common endocrine disease in the world. DM is a chronic inflammatory disease characterized by high blood glucose levels. Low grade inflammation and immune activation are closely related to the pathogenesis and complications of DM (1).

Diabetic foot syndromes (ulcers) cause decrease in quality of life in diabetic patients. This syndrome is the most common cause of hospitalization and surgery in patients with DM and has a high morbidity and mortality rate. For example, $85 \%$ of foot amputations are secondary to diabetic foot ulcers (DFU) (2).
The balance between pro- and anti-inflammatory processes is impaired in patients with DFU (3). The most common procedure for diagnosis of DFU is evaluating physical changes such as skin irritation, pain, fever and redness in the foot region $(4,5)$. Also, culturing the sample taken from patient's foot is applied to observe pathogen proliferation (4). As the infected area enlarges, other symptoms are promoted. There is no optimal treatment for diabetic foot most of the treatment modalities are very expensive and it is not easy to control the disease (6). Diagnostic process is the key point for determining the route of treatment.

Interleukin (IL)-12 P70 is a cytokine with an important role in the initiation of native and adaptive immune responses to 
many infections (7). IL-12 P70 is a heterodimer cytokine and is released from macrophages, dendritic cells, langerhans cells, $\mathrm{B}$ cells, and natural killer cells. It plays an important role in cell-mediated immunity. Production of IL-12 P70 is increased in viral, fungal and bacterial infections. IL-12 P70 contributes to the production and maturation of natural killer cells and pro-inflammatory cytokines such as IL-2, IL-3, and tumor necrosis factor- $\alpha$ (8). There are some studies showing that IL12 P70 plays a role in pathogenesis of DM type 1 and $2(9,10)$. IL-17 is an important pro-inflammatory cytokine. It increases chemokine production and organizes monocytes and neutrophils (11). IL-17 has been shown to play a role in autoimmune diseases such as inflammatory bowel disease, psoriasis, systemic lupus erythematous, allergy, asthma and multiple sclerosis (12). Emamaullee et al. (13) performed a study on non-obese diabetic mice and they showed that IL-17 is involved in pathogenesis and plays an important role in the fight against extracellular pathogens such as Candida and Klebsiella. IL-18, previously known as interferon- $\gamma$-inducing factor, is an immune-regulatory cytokine secreted from activated macrophages and first described in 1989 (14). IL-18 is secreted by monocytes, macrophages and osteoclasts in osteoblasts, epithelial cells, keratinocytes, adrenal cortex, and the pituitary gland, endometrium and ovary $(15,16)$.

Recently, Tuttolomondo et al. (17-19) showed that IL-1, IL-6 and resistin levels in plasma can be used as markers for determining type 2 diabetes with and without diabetic foot syndrome and they found that there was a relation between cardiovascular morbidity and inflammatory cytokines in patients with diabetic foot syndromes.

Skin irritation, symptoms such as redness, pain and fever around the foot region, some imaging methods such as radiograph and magnetic resonance imaging, C-reactive protein level, leucocyte count, erythrocyte sedimentation rate and related tests are all used in diagnosis of DFU currently $(5,19,20)$.

In the literature, there are different ILs evaluated as a marker for diagnosis of patients with DM and DFU. However, there are not conspicuous studies upon IL-12 P70, IL-18 and IL-17, even though DM and DFUs are related with inflammation as IL-12 P70, IL-17 and IL-18 are. The aim of the present study was to investigate serum IL-12 P70, IL-17, IL-18 levels and some biochemical parameters in subjects with type $2 \mathrm{DM}$ and diabetic foot syndromes, and healthy controls, and to demonstrate a relationship between IL parameters and type $2 \mathrm{DM}$ and diabetic foot syndromes.

\section{Methods}

The study was carried out in the Faculty of Medicine of Sakarya University. The patients were provided by the department of internal medicine. The study was approved by the Ethics Committee of Faculty of Medicine of Sakarya University and informed consent was obtained from every subject. An application was made to institutional ethical committee on 22.05.2013 for the study. The study was started after decision dated 18.06.2013 and numbered 71522473.050.01.04/41 from the Ethics Committee of Faculty of Medicine of Sakarya University.

\section{Subjects}

This study included 95 participants: 31 with DFU (group 1), 33 with type $2 \mathrm{DM}$ (group 2) and 31 healthy subjects as a control group (group 3). Group 1 was consisted of 21 males and 10 females aged 51-80 (61.2 \pm 8.35$)$ years. Group 2 was consisted of 19 males and 14 females aged $42-75(60.4 \pm 8.81)$ years and group 3 was consisted of 17 males and 14 females aged 51- 80 $(61.2 \pm 8.35)$ years.

Patients with malignancy, pregnancy, cirrhosis, renal failure, or class 3 or 4 heart failure according to the New York Heart Association (NYHA) classification, systemic inflammatory diseases, undergoing immunosuppressive therapy, Wagner grade 4 and 5 diabetic ulcers, or thrombosis were excluded from the study. The presence of purulent secretions or the presence of two or more signs of inflammation (e.g., erythema, warmth, tenderness, heat, and induration) were regarded as evidence of infection. The condition was described in a consensus development conference on diabetic foot wound care (20).

Patients with 18-80 years, type 2 DM, presence of purulent secretions, presence of two or more signs of inflammation, patients who were not pregnant, did not have cancer, did not undergo immunosuppressive therapy, and who did not have class 3 or 4 heart failure according to the NYHA were involved in this study.

\section{Samples}

Blood samples were taken from all groups in tubes containing ethylenediamine tetraacetic acid (EDTA) and non-EDTA after overnight fasting. Blood samples were taken in non-EDTA tubes for human IL-12 P70, IL-17, IL-18, glucose, total cholesterol, low-density lipoprotein cholesterol (LDL-C), triglyceride-C, high-density lipoprotein cholesterol (HDL-C), and urea and creatinine analysis, then centrifuged at $+4{ }^{\circ} \mathrm{C} 3000 \mathrm{rpm}$ for 10 min and sera were immediately separated from the cells after centrifugation. Serum samples for the measurement of human IL-12 P70, IL-17, and IL-18 were stored at $-80{ }^{\circ} \mathrm{C}$ until being used. All measurements were done in the same series after the samples were thawed. Blood samples taken in EDTA tubes for hematological tests, and hemoglobin $(\mathrm{Hb}) \mathrm{A} 1 \mathrm{c}$ parameters were studied at the same day in routine laboratory.

\section{Biochemical Parameters}

HbA1c, glucose, total cholesterol, LDL-C, triglyceride-C, HDL-C, urea and creatinine analysis and hematological tests were measured using commercial kits in routine biochemistry laboratory. HbAlc from Trinity Biotech (Trinity Biotech Assay and System, USA), glucose, total cholesterol, triglyceride-C, urea and creatinine from Abbott Architect (fully automatic analyzer, Architect System c1600; Abbott Laboratories, Lake Bluff, IL, USA), LDL-C and HDL-C from Archem Diagnostics for Abbott Architect, USA were used. Hematology parameters were measured using the Cell-Dyne 3700 SL hematology analyzer (Abbott Laboratories, North Chicago, IL, USA). 


\section{ELISA Assay}

Human IL-12 P70, IL-17 and IL-18 levels were measured with an enzyme-linked immunosorbent assay kit (reference: BMS 238, lot number: 95350039; reference: BMS 2017, lot number: 96656015 ; reference: BMS 267/2, lot number: 96934032 respectively; ELISA kits manufactured for eBioscience by Bender MedSystems GmbH Campus Vienna Biocenter 2, 1030 Vienna, Austria, www. eBioscience.com). All ELISA assays were performed via an ELISA autoanalyzer (BioTek 800 TS Absorbance Reader, USA).

\section{Statistical Analysis}

Descriptive analyses were performed to provide information on general characteristics of the study population. KolmogorovSmirnov test was used to evaluate whether the distribution of continuous variables were normal. ANOVA or Kruskal-Wallis $\mathrm{H}$ test was used to compare the continuous data among groups. For multiple comparison of ANOVA; Tukey or Tamhane T square was used. Beside for multiple comparison of Kruskal-Wallis $\mathrm{H}$ test, Dunn's test was used. The continuous data were presented as the mean \pm standard deviation. Receiver operating characteristics (ROC) curve analysis was used to determine the cut off value of IL-12 P70, 17 and 18 levels in diagnosis of type 2 DM with diabetic foot. Sensitivity and specificity of the test as well as the positive and negative predictive values were calculated for IL12 P70, 17 and 18. A p value $<0.05$ was considered significant. Analyses were performed using commercial software (IBM SPSS
Statistics, version 23.0; Armonk, NY: IBM Corp.). ROC curves were constructed using MedCalc (MedCalc Statistical Software version 15.8, MedCalc Software bvba, Ostend, Belgium).

\section{Results}

The levels of HbA1c, glucose, total cholesterol, LDL-C, triglyceride, HDL-C, urea and creatinine were shown in Table 1.

As seen in Table 1, no statistically significant difference was found in terms of HbA1c and fasting glucose between group 1 and group $2(p>0.05)$. However it was found that there were significant differences between group 1 and group 3 and between group 2 and group $3(\mathrm{p}<0.05)$ for same variables. No statistically significant difference was found in levels of LDL-C, HDL-C, triglyceride, total cholesterol, urea and creatinine between group 1 and group 2 ( $p>0.05)$. However it was found that there were significant differences between group 1 and group 3, between group 2 and group 3 for LDL-C, and also between group 2 and group 3 for total cholesterol $(\mathrm{p}<0.05)$.

As seen in Table 2, the highest levels of IL-12 P70, IL-17 and white blood cell were found in group 1. These levels were significantly higher in group 1 than in group $3(\mathrm{p}<0.05)$, but there was not significant difference between group 1 and group 2 $(\mathrm{p}>0.05)$. IL-18 levels in group 1 and group 2 were significantly higher than group $3(\mathrm{p}<0.05)$, but there was no significant difference between group 1 and group 2 ( $p>0.05)$.

\section{Table 1. Comparison of the biochemical parameters among groups}

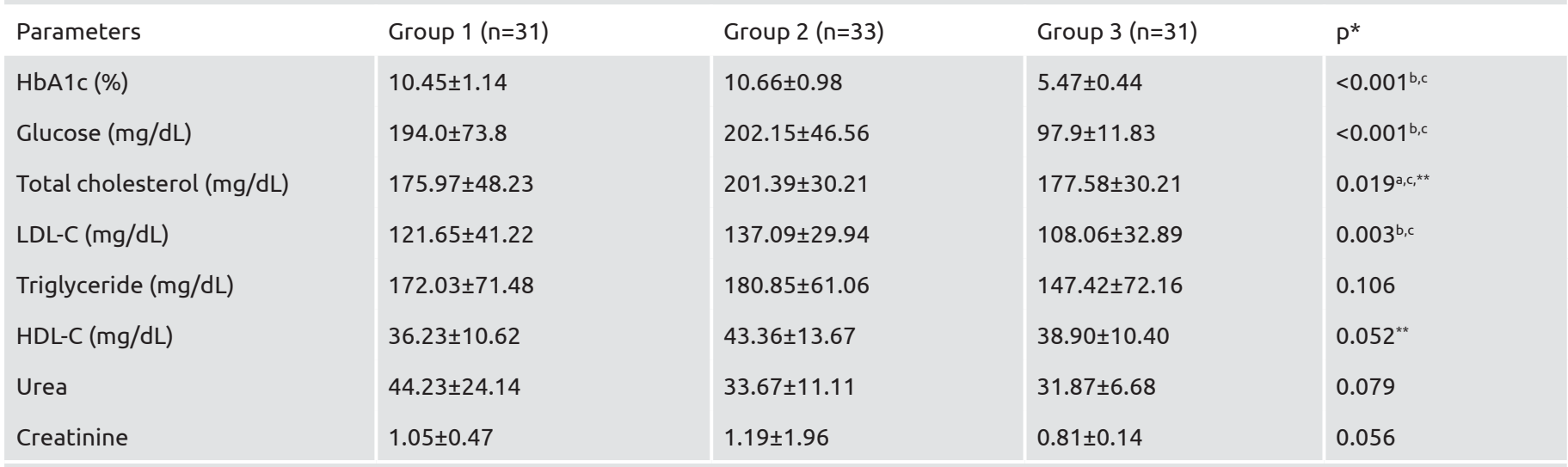

HbA1 c: hemoglobin A1C; LDL-C: low-density lipoprotein cholesterol; HDL-C: high-density lipoprotein cholesterol; group 1: diabetic foot syndromes; group 2: diabetes mellitus type 2; group 3: control

*: results of the Kruskal-Wallis $\mathrm{H}$ test; **: results of the ANOVA; a: there is a statistically significant difference between group 1 and 2 ; b: there is a statistically significant difference between group 1 and $3 ;$; : there is a statistically significant difference between group 2 and 3 ; data were shown as mean \pm standard deviations

Table 2. Comparison of the inflammatory parameters among groups

\begin{tabular}{|c|c|c|c|c|}
\hline Parameters & Group $1(n=31)$ & Group 2 (n=33) & Group 3 (n=31) & $p^{*}$ \\
\hline WBC $\left(10^{3} / \mu \mathrm{L}\right)$ & $10.07 \pm 5.38$ & $7.88 \pm 3.41$ & $6.03 \pm 1.45$ & $<0.001^{\mathrm{a}, \mathrm{b}}$ \\
\hline IL-12 P70 (pg/mL) & $13.61 \pm 12.87$ & $10.80 \pm 10.58$ & $6.89 \pm 5.83$ & $0.007^{b}$ \\
\hline IL-17 (ng/mL) & $7.15 \pm 6.99$ & $5.87 \pm 7.05$ & $2.50 \pm 1.04$ & $<0.001^{b, c}$ \\
\hline IL-18 (pg/mL) & $464.84 \pm 279.39$ & $483.56 \pm 232.08$ & $280.29 \pm 151.87$ & $<0.001^{\mathrm{b}, \mathrm{c}}$ \\
\hline
\end{tabular}


The performances of IL-12 P70, 17 and 18 biomarkers in diagnosing DFU and in separating it from healthy controls were statistically significant [respectively; area under the curve $(\mathrm{AUC})=0.736,0.791$ and $0.723 ; \mathrm{p}<0.001, \mathrm{p}<0.001, \mathrm{p}<0.001]$. Cut-off values for relevant variables were calculated as $>5.42$, $>3.3,>407.2$, respectively (Table 3 and Figure 1).

The performances of IL-12 P70, 17 and 18 in diagnosing type 2 $\mathrm{DM}$ and in separating it from healthy controls were statistically

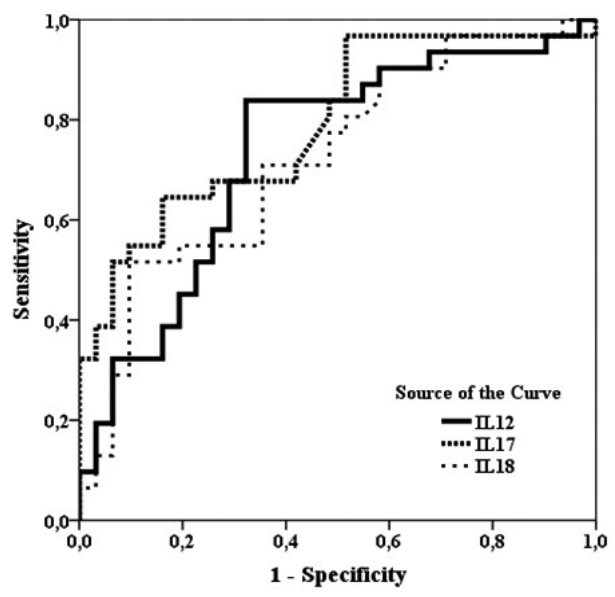

Figure 1. Receiver operating characteristics curves of the interleukin-12 P70, 17 and 18 in diagnosing diabetes mellitus type 2 with diabetic foot ulcer group and in separating it from control group

IL: interleukin significant, but no statistically significant difference was found in terms of IL-17 (respectively; AUC $=0.656,0.678$ and 0.785, $\mathrm{p}=0.030, \mathrm{p}=0.085$ and $\mathrm{p}<0.001)$. Cut-off values for relevant variables were calculated as $>5.9,>1.98,>315.58$, respectively (Table 4 and Figure 2).

In diagnosing diabetic foot according to DM type 2 group, the performances of IL-12 P70, 17 and 18 in diagnosis of DFU and in separating it from type $2 \mathrm{DM}$ were not statistically significant,

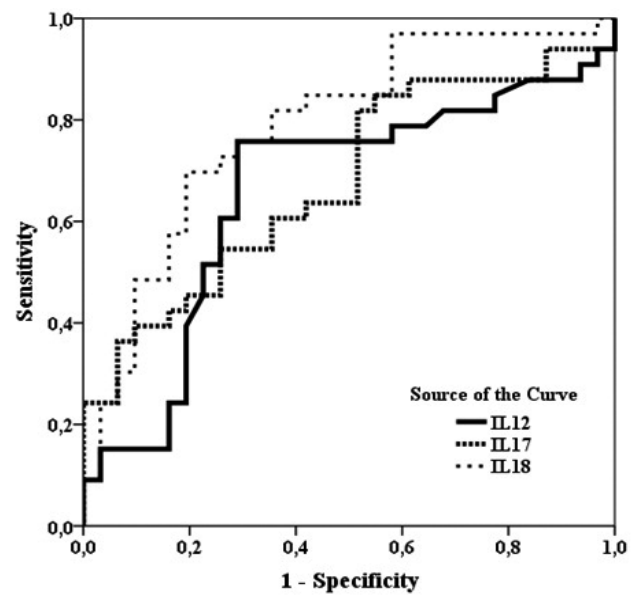

Figure 2. Receiver operating characteristics curves of the interleukin-12 P70, 17 and 18 in diagnosing diabetes mellitus type 2 group and separating it from control group IL: interleukin

Table 3. Diagnostic performances of interleukin-12 P70,17 and 18 in diagnosing diabetes mellitus type 2 with diabetic foot ulcer group and in separating it from control group

\begin{tabular}{|c|c|c|c|c|c|c|c|c|c|}
\hline \multirow{2}{*}{ DTOMalkets } & \multirow{2}{*}{\multicolumn{2}{|c|}{ Cul-oir values }} & \multirow[t]{2}{*}{ SE OI AU } & \multirow[t]{2}{*}{ 95\% С } & \multirow[t]{2}{*}{ P values } & \multirow[t]{2}{*}{ sems. } & \multirow[t]{2}{*}{ spec. } & \multirow{2}{*}{ PPV } & \multirow[b]{2}{*}{ NPV } \\
\hline & & & & & & & & & \\
\hline IL-12 P70 & $>5.42$ & 0.736 & 0.0653 & 0.608 to 0.840 & $<0.001$ & 83.87 & 67.74 & 72.2 & 80.8 \\
\hline IL-17 & $>3.3$ & 0.791 & 0.0576 & 0.669 to 0.884 & $<0.001$ & 64.52 & 83.87 & 80.0 & 70.3 \\
\hline IL-18 & $>407.2$ & 0.723 & 0.0652 & 0.594 to 0.829 & $<0.001$ & 51.61 & 90.32 & 84.2 & 65.1 \\
\hline
\end{tabular}

AUC: area under the curve; SE: standard error; Cl: confidence interval; IL: interleukin; Sens.: sensitivity; Spec.: specificity; PPV: positive predictive value; NPV: negative predictive value

Table 4. Diagnostic performances of interleukin-12 P70, 17 and 18 in diagnosing diabetes mellitus type 2 group and separating it from control group

\begin{tabular}{|c|c|c|c|c|c|c|c|c|c|}
\hline \multirow{2}{*}{ Biomarkers } & \multirow{2}{*}{ Cut-off values } & \multirow{2}{*}{ AUC } & \multirow{2}{*}{ SE of AUC } & \multirow{2}{*}{$95 \% \mathrm{Cl}$ of AUC } & \multirow{2}{*}{$p$ values } & \multirow{2}{*}{ Sens. } & \multirow{2}{*}{ Spec. } & \multicolumn{2}{|c|}{ Predictive values (\%) } \\
\hline & & & & & & & & PPV & NPV \\
\hline IL-12 P70 & $>5.9$ & 0.656 & 0.0722 & 0.527 to 0.771 & 0.030 & 75.6 & 70.97 & 73.5 & 73.3 \\
\hline IL-17 & $>1.98$ & 0.678 & 0.0678 & 0.550 to 0.790 & 0.085 & 81.82 & 48.39 & 62.8 & 71.4 \\
\hline
\end{tabular}


Table 5. Diagnostic performances of interleukin-12 P70,17 and 18 in diagnosing diabetes mellitus type 2 with diabetic foot ulcer group and in separating it from diabetes mellitus type 2 group

\begin{tabular}{|c|c|c|c|c|c|c|c|c|c|}
\hline \multirow{3}{*}{ Biomarkers } & \multirow{3}{*}{ Cut-off values } & \multirow{3}{*}{ AUC } & \multirow{3}{*}{ SE of AUC } & \multirow{3}{*}{$95 \% \mathrm{Cl}$ of $\mathrm{AUC}$} & \multirow{3}{*}{$p$} & \multirow{3}{*}{ Sens. } & \multirow{3}{*}{ Spec. } & \multicolumn{2}{|c|}{ Predictive values (\%) } \\
\hline & & & & & & & & & \\
\hline & & & & & & & & PPV & NPV \\
\hline IL-12 P70 & $>10.38$ & 0.542 & 0.0741 & 0.412 to 0.667 & 0.575 & 41.94 & 75.76 & 61.9 & 58.1 \\
\hline IL-17 & $>3.46$ & 0.610 & 0.0714 & 0.480 to 0.729 & 0.124 & 64.52 & 60.61 & 60.6 & 64.5 \\
\hline IL-18 & $\leq 295.4$ & 0.556 & 0.0741 & 0.427 to 0.680 & 0.448 & 45.16 & 78.79 & 66.7 & 60.5 \\
\hline
\end{tabular}

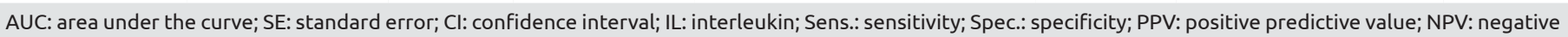
predictive value

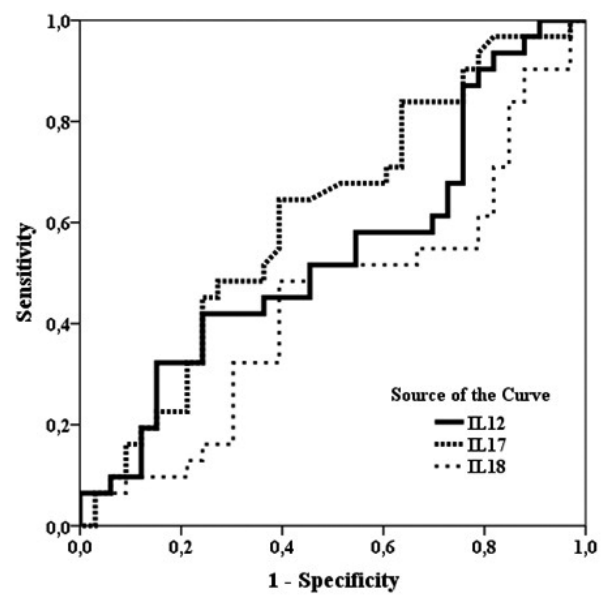

Figure 3. Receiver operating characteristics curves of the interleukin-12 P70, 17 and 18 in diagnosing diabetes mellitus type 2 with diabetic foot ulcer group and in separating it from diabetes mellitus type 2 group IL: interleukin

(respectively; $\mathrm{AUC}=0.542,0.610,0.556$ and $\mathrm{p}=0.575, \mathrm{p}=0.124$, $\mathrm{p}=0.448)$. Cut-off values for relevant variables were calculated as $>5.9,>1.98,>315.58$, respectively (Table 5 and Figure 3 ).

\section{Discussion}

Our study demonstrated a relationship between serum IL-12 P70, IL-17, and IL-18 levels and both DM type 2 and DFU.

It was reported that the frequency of cardiovascular diseases increases 2-4 times in diabetic patients with DFU (19). The comparison of biochemical parameters such as LDL, triglyceride and total cholesterol levels between normal and diabetic individuals shows that there is correlation between inflammation and DM (19). Recent studies indicated that the level of inflammatory cytokines IL-1, IL-6 were higher in diabetic patients with cardiovascular diseases (18).

It was reported that cardiovascular diseases are more common in diabetic patients compared with non-diabetic patients (21). Recent studies indicated that the level of inflammatory cytokines IL-1, IL-6 were higher in diabetic patients with cardiovascular diseases (19). However, there is not much study on IL-12 P70, IL-17, and IL-18 levels in diabetic patients.
Studies about DM type 2 and IL-12 P70 were found in the literature $(6,7)$. IL-12 P70 is a pro-inflammatory cytokine which stimulates the proliferation and migration of $\mathrm{T}$ cells and natural killer cells (22). Type $2 \mathrm{DM}$ is a chronic inflammatory disease $(23,24)$. High IL-12 P70 levels in patients with type 2 DM are associated with pro-inflammatory cytokines and chronic inflammatory disease. Our results were compatible with this mechanism and the literature.

Development of DFU is multifactorial. Diabetic foot ulcerations occur as a result of complex interactions of factors such as peripheral artery disease, atherosclerotic plaque, changes in the microcirculation, and peripheral neuropathy. Proliferation and migration of T-cell and natural killer cells induced by IL-12 P70 play an important role in the formation of atherosclerotic plaques (25). Atherosclerotic plaques can lead to changes in microcirculation and peripheral arterial disease (26), as well as the production of IL-12 P70 is increased in viral, fungal, and bacterial infections (8). The role of IL-12 P70 in DFU can be explained by the increase in the reproducing rate of bacteria in diabetic ulcer's areas. In our study, the highest levels of IL-12 P70 were found in the DFU group. We think that these levels can be explained in two ways: 1) IL-12 P70 is a pro-inflammatory cytokine, 2) IL-12 P70 can play a role in the development of diabetic foot syndrome.

However, there is not enough data about IL-17, and IL-18 levels in patients with DM in the literature. IL-17 is a pro-inflammatory cytokine released by $\mathrm{T}$ cells. There are some studies and animal models which show that IL-17 plays a role in the pathogenesis of DM type 1 . Type $1 \mathrm{DM}$ was prevented in the non-obese mice with anti-IL-17 treatment (10). The high presence of IL-17 levels in these studies are related with autoimmune diseases. IL17 has also been implicated in allergic skin response (27). Gramnegative bacteria such as Escherichia coli, Pseudomonas aeruginosa, and Klebsiella spp. are more common in severe DFU (28). IL-17 plays an important role in fighting against extracellular pathogens such as Candida and Klebsiella (12). According to our results, we think that high IL-17 levels in both DFU and DM groups may be attributed to the fact that IL-17 is a pro-inflammatory cytokine. High IL-17 levels in patients with DFU may be due to inflammation in the ulceration, deterioration of skin integrity, and various types of bacteria causing infections. In our study, higher level of IL-17 in both DFU and DM groups compared 
with control group gives an idea that IL-17 may be related with diabetic disease and can be used as a marker in diagnosis of DM. Additionally, higher level of IL-17 in DFU group suggests that IL-17 can be used as a pro-inflammatory marker for diabetic foot.

IL-18 is a pro-inflammatory cytokine secreted by epithelial cells, macrophages and dendritic cells; and known as an interferon- $\gamma$ stimulating factor (14). Elevated IL-18 levels are an independent predictor of type $2 \mathrm{DM}$ and metabolic syndrome (29). In an animal study, IL-18 has been shown to cause $\beta$-cell dysfunction and deterioration of insulin secretion (30). IL-18 causes endothelial dysfunction and plays a role in atherosclerosis (31). In another study, the relationship between IL-18 and DFU was examined. IL-18 is a pro-inflammatory cytokine (32). A study showed the correlation between plasma IL-18 levels and plasma fasting glucose; plasma IL-18 levels and insulin levels; plasma IL-18 levels and obesity in females; and plasma IL-18 levels and DFU (16). In our study, high levels of IL-18 in the patients with type $2 \mathrm{DM}$ were consistent with the literature. High levels in patients with DFU are due to the role of IL-18 in atherosclerosis, as IL-18 is pro-inflammatory cytokine. Higher level of IL-18 in both DFU and DM groups suggests IL-18 may be used as a marker in diagnosis of them.

Studies have shown that high LDL level and low HDL-C in type 2 DM patients are related with cardiovascular diseases (33). In our study, higher level of LDL in both patients with type 2 DM and patients with diabetic foot syndrome was supported by these studies. According to the AHEAD Research Group's study, intensive lifestyle intervention or diabetes support and education may help to increase HDL-C level in diabetic patients (33). Nevertheless, there was no significant difference in terms of HDL-C between diabetic foot syndrome, DM and control groups in our study.

HbA1c is the best indicator for glycemic control. It is used as significant parameter to monitor blood glucose level in patients with DM (34). We found high level of HbAlc in patients with type $2 \mathrm{DM}$ and diabetic foot syndrome in our study, which were expected.

\section{Study Limitations}

This study was conducted within a limited period. For this reason, it is considered that increasing the number of patients and studying other IL inflammation markers may be appropriate.

\section{Conclusion}

We think that IL-12 P70, 17 and 18 parameters can be used as biomarkers for diagnosis of DFU and type $2 \mathrm{DM}$. This study will guide researchers for future studies to find inflammatory biomarkers in DM and DFU. According to our results, further comprehensive studies are needed to investigate specific biomarkers for diagnosis of DFU related with inflammatory.

\section{Ethics}

Ethics Committee Approval: This study was approved by the Ethics Committee of Faculty of Medicine of Sakarya University, dated 18.06.2013 and numbered 71522473.050.01.04/41.
Informed Consent: Written informed consent was obtained from patients who participated in this study.

Peer-review: Externally peer-reviewed.

\section{Authorship Contributions}

Concept: S.K., C.V., A.N., Design: S.K., C.V., Data Collection or Processing: S.K., C.V., A.N., Analysis or Interpretation: S.K., C.V., A.N., H.Y., M.A., Literature Search: S.K., C.V., Writing: S.K., C.V.

Conflict of Interest: The authors have no conflicts of interest to declare.

Financial Disclosure: This study was funded by Scientific Research Projects Unit in Sakarya University (the number of project: 2013-0806009).

\section{References}

1. Navarro-Gonzalez JF, Mora-Fernandez C. The role of inflammatory cytokines in diabetic nephropathy. J Am Soc Nephrol 2008;19:43342.

2. The Expert Committee on the diagnosis and classification of diabetes mellitus. Report of the Expert Committee on the diagnosis and classification of diabetes mellitus. Diabetes Care 1997;20 (Suppl 1):1183-97.

3. Weigelt C, Rose B, Poschen U, Ziegler D, Friese G, Kempf K, et al. Immune mediators in patients with acute diabetic foot syndrome. Diabetes Care 2009;32:1491-6.

4. Lipsky BA, Berendt AR, Deery HG, Embil JM, Joseph WS, Karchmer AW, et al. Diagnosis and Treatment of Diabetic Foot Infections. Clin Infect Dis 2004;39:885-910.

5. Alavi A, Sibbald RG, Mayer D, Goodman L, Botros M, Armstrong DG, et al. Diabetic foot ulcers: part II. Management. J Am Acad Dermatol 2014; 70: 21.e1-24; quiz 45-6.

6. Moura LI, Dias AM, Carvalho E, de Sousa HC. Recent advances on the development of wound dressings for diabetic foot ulcer treatment: A review. Acta Biomater 2013;9:7093-114.

7. Schulz O, Edwards AD, Schito M, Aliberti J, Manickasingham S, Sher A, et al. CD40 triggering of heterodimeric IL-12 P70 production by dendritic cells in vivo requires a microbial priming signal. Immunity 2000;13:453-62.

8. Trinchieri G. Function and clinical use of interleukin-12. Curr Opin Hematol 1997;4:59-66.

9. Stern AS, Preskey DH. Interleukin 12-an integral cytokine in the immune response. Life Sci 1996;58:639-54.

10. Skarsvik S, Ludvigsson J, Vaarala O. Aberrant regulation of interleukin-12 receptor 2 chain on type 1 cytokine stimulated T lymphocytes in type 1 diabetes. Immunology 2005;114:287-93.

11. Ke Y, Liu K, Huang GQ, Cui Y, Kaplan HJ, Shao H, et al. Antiinflammatory role of IL-17 in experimental autoimmune uveitis. J Immunol 2009;182:3183-90.

12. Maddur MS, Miossec P, Kaveri SV, Bayry J. Th17 cells: biology, pathogenesis of autoimmune and inflammatory diseases, and therapeutic strategies. Am J Pathol 2012;181:8-18. 
13. Emamaullee JA, Davis J, Merani S, Toso C, Elliott JF, Thiesen A, et al. Inhibition of Th17 cells regulates autoimmune diabetes in NOD mice. Diabetes 2009;58:1302-11.

14. Okamura H, Tsutsi H, Komatsu T, Yutsudo M, Hakura A, Tanimoto $\mathrm{T}$, et al. Cloning of a new cytokine that induces IFN-gamma production by T cells. Nature 1995;378:88-91.

15. Dinarello CA, Novick D, Puren AJ, Fantuzzi G, Shapiro L, Mühl $\mathrm{H}$, et al. Overview of interleukin 18; more than an interferon gama inducing factor. J Leukoc Biol 1998;63:658-64.

16. Oikawa Y, Shimada A, Kasuga A, Morimoto J, Osaki T, Tahara H, et al. Systemic administration of IL-18 promotes diabetes development in young nonobese diabetic mice. J Immunol 2003;171:5865-75.

17. Tuttolomondo A, Casuccio A, Guercio G, Maida C, Del Cuore A, Di Raimondo D, et al. Arterial stiffness, endothelial and cognitive function in subjects with type 2 diabetes in accordance with absence or presence of diabetic foot syndrome. Cardiovasc Diabetol 2017;16:2.

18. Tuttolomondo A, La Placa S, Di Raimondo D, Bellia C, Caruso A, Lo Sasso B, et al. Adiponectin, resisting and IL-6 plasma levels in subjects with diabetic foot and possible correlations with clinical variables and cardiovascular co-morbidity. Cardiovasc Diabetol 2010;9:1-2.

19. Tuttolomondo A, Maida C, Pinto A. Diabetic foot syndrome: Immune-inflammatory features as possible cardiovascular markers in diabetes. World J Orthop 2015;6:62-76.

20. Lipsky BA. Osteomyelitis of the foot in diabetic patients. Clin Infect Dis 1997;25:1318-26.

21. Smith JW, Marcus FI, Serokman R. Prognosis of patients with diabetes mellitus after acute myocardial infarction. Am J Cardiol 1984;54:718-21.

22. Herder A, Haaster B, Muller-Scholze S, Koening W, Thorand B, Holle R, et al. Association of systemic chemokine concentrations with impaired glucose tolerance and type 2 diabetes: results from the Cooperative Health Research in the Region of Augsburg Survey S4 (KORA S4). Diabetes 2005;54:11-7.

23. Tataranni PA, Ortega EA. A burning question: Does adipokineinduced activation of the immune system mediate the effect of over nutrition on type 2 diabetes? Diabetes 2005;54:917-27.

24. Fei GZ, Huang YH, Swedenborg J, Frostegard J. Oxidised LDL modulates immune-activation by an IL-12 P70 dependent mechanism. Atherosclerosis 2003;169:77-85.
25. Brownrigg JR, Apelqvist J, Bakker K, Schaper NC, Hinchliffe RJ. Evidence-based management of PAD \&amp; the diabetic foot. Eur J Vasc Endovasc Surg 2013;45:673-81.

26. Albanesi C, Cavani A, Girolomoni G. IL-17 Is Produced by NickelSpecific T Lymphocytes and Regulates ICAM-1 Expression and Chemokine Production in Human Keratinocytes: Synergistic or Antagonist Effects with IFN- $\gamma$ and TNF- $\alpha$. J Immunol 1999;162:494-502.

27. Pathare NA, Bal A, Talvalkar GV. Antani DU. Diabetic foot infections: A study of microorganisms associated with the different Wagner grades. Indian J Pathol Microbiol 1998;41:437-41.

28. Esposito K, Nappo F, Giugliano F, Di palo C, Ciotola M, Barbieri M, et al. Meal modulation of circulating interleukin 18 and adiponectin concentrations in healthy subjects and in patients with type 2 diabetes mellitus. Am J Clin Nutr 2003;78:1135-40.

29. Fischer CP, Perstrup LB, Bernsten A, Eskildsen P, Pedersen BK. Elevated plasma interleukin-18 is a marker of insulin-resistance in type 2 diabetic and non-diabetic humans. Clin Immunol 2005;117:152-60.

30. Frigerio S, Hollander GA, Zumsteg U. Functional IL-18 Is produced by primary pancreatic mouse islets and NIT-1 beta cells and participates in the progression towards destructive insulitis. Horm Res 2002;57:94-104.

31. Gerdes N, Sukhova GK, Libby P. Expression of interleukin (IL)-18 and functional IL-18 receptor on human vascular endothelial cells, smooth muscle cells, and macrophages: implications for atherogenesis. J Exp Med 2002;195:245-57.

32. Sabuncu T, Eren MA, Tabur S, Dag OF, Boduroglu O. High serum concentration of interleukin-18 in diabetic patients with foot ulcers. J Am Podiatr Med Assoc 2014;104:222-6.

33. The Look AHEAD Research Group. Long Term Effects of a Lifestyle Intervention on Weight and Cardiovascular Risk Factors in Individuals with Type 2 Diabetes: Four Year Results of the Look AHEAD Trial. Arch Intern Med 2010;170:1566-75.

34. The Diabetes Control and Complications Trial Research Group. The effect of intensive treatment of diabetes on the development and progression of long-term complications in insulin-dependent diabetes mellitus. N Eng J Med 1993;329:977-86. 